\title{
Etude clinique évaluant les effets du bevacizumab (Avastin) sur la sévérité des ostéonécroses des maxillaires associées à l'acide zolédronique chez les patients cancéreux.
}

\author{
Lescaille G, Coudert A, Baaroun V, Ostertag A, Charpentier E, Javelot MJ, Toledo R, Goudot P, Azerad J, Berdal A, Spano JP, Ruhin B, \\ Descroix V \\ Service d'Odontologie, Groupe Hospitalier Pitié-Salpêtrière, 47-83 Boulevard de l'Hôpital, 75013, Paris, FRANCE
}

Les ostéonécroses des maxillaires sont des complications connues des bisphosphonates notamment dans des indications oncologiques, et lorsqu'ils sont administrés en IV. II a été par ailleurs montré que le Bevacizumab (BVZ) pourrait également être à l'origine d'ostéonécroses des maxillaires (ONM). En outre, le BVZ est indiqué dans le traitement de certains cancers y compris métastatiques, et peut donc être associé à la prise de bisphosphonates. L'objectif de cette étude était d'évaluer les effets du BVZ sur la sévérité des ONM dans une cohorte de patients cancéreux traités par acide zolédronique (AZ). Nous avons analysé les données de 42 patients atteints d'ONM entre 2007 et 2010. Seuls les patients traités par AZ pour des cancers solides avec métastases osseuses ont été inclus. Nous avons donc volontairement exclu les patients atteints de myélome, notamment du fait des traitements de chimiothérapie très différents, et l'absence d'indication de BVZ chez ces patients. Les données analysées ont été l'âge, le sexe, les comorbidités, les antécédents dentaires et les caractéristiques des ONM. Sur les 42 patients traités par AZ, 10 ont reçu également du BVZ. Dans le groupe des 10 patients traités par AZ/BVZ, la durée moyenne de traitement par $A Z$ au diagnostic d'ONM était de 12.4 mois $( \pm 6.8)$, comparé à 22.9 mois $( \pm 4.8)$ chez 32 patients qui n'ont pas reçu de BVZ en association à l'AZ $(p<0.05)$. L'analyse du modèle de COX confirme l'impact du BVZ sur le délai diagnostic de l'ostéonécrose. Dans le groupe AZ/BVZ, $7(70 \%)$ des patients ont développé une ostéonécrose spontanée. L'analyse multivariée montre que l'association AZ/BVZ augmente le risque de développer une ostonécrose spontanée (OR 6.07; 95\% $\mathrm{Cl}$, [1.3 - 28.2], $\mathrm{p}<0.05)$. Par ailleurs, le nombre de localisations d'ostéonécrose était augmenté dans le groupe AZ/BZV comparé au groupe traité par $A Z(p<0.01)$. D'autres paramètres cliniques comme le type de tumeur (prostate, sein..), la sévérité du cancer ou encore certains types de chimiothérapies peuvent également avoir un rôle dans le développement de ces ostéonécroses. Cette étude démontre pour la première fois l'impact négatif du BVZ dans l'incidence et la sévérité des ONM traités par ZA. Nos résultats suggèrent que la combinaison AZ/BVZ prédispose au développement d'ONM précoce et spontanée.

LESCAILLE Géraldine geraldine.lescaille@gmail.com

This is an Open Access article distributed under the terms of the Creative Commons Attribution License 4.0, which permits unrestricted use, distribution, and reproduction in any medium, provided the original work is properly cited. 